\title{
Les Loranthaceae et Viscaceae parasites des forêts et des agro-écosystèmes du Burundi : diversité et ampleur d'infestation
}

\author{
Habonimana Bernadette. (1,*), Nzigidahera Benoît. (2).†, Hakizimana Paul. (3)., Ndayisaba Gilbert. (1) et \\ Masharabu Tatien $(3, *)$ \\ (1) Université du Burundi, Faculté d'Agronomie et de Bio-Ingénierie, B.P. 2940 Bujumbura, Burundi \\ (2) Office Burundais pour la Protection de l'Environnement (OBPE), B.P. 56 Gitega, Burundi \\ (3) Université du Burundi, Faculté des Sciences, Département de Biologie, B.P. 2700 Bujumbura, Burundi \\ (*)Auteur(s) pour correspondance : habonimanaberna@gmail.com \& tatien.masharabu@ub.edu.bi
}

Original submitted in on 21st May 2019. Published online at www.m.elewa.org/journals/ on $31^{\text {st }}$ August 2019 https://dx.doi.org/10.4314/jab.v140i1.3

\section{RÉSUMÉ}

Objectif : Cette étude visait à inventorier les hémi-parasites Loranthaceae et Viscaceae occasionnant des dégâts sur les arbres et arbustes des forêts et des milieux agricoles du Burundi ainsi que leur ampleur d'infestation.

Méthodologie et Résultats : L'étude a été conduite dans toutes les zones agro-écologiques du pays dans les forêts et les milieux agricoles environnants. Après prospection, 98 sites répartis sur 192 ha ont été retenus sur base de l'abondance des individus parasités. Au total, 15 espèces hémi-parasites ont été inventoriées dont 11 Loranthaceae et 4 Viscaceae. La crête Congo-Nil présente la plus grande diversité avec 8 espèces sur les 15 tandis que la plaine de l'Imbo et la dépression du Kumoso ne comptent que 2 espèces chacune. Nonante cinq espèces hôtes réparties dans 64 genres et 37 familles ont été recensées dont $47 \%$ sont infestées par Phragmanthera usuiensis. Concernant l'ampleur d'infestation, le taux le plus élevé se rencontre dans la dépression du Kumoso tandis que l'intensité d'infestation est la plus forte dans la dépression du Nord-Est. Elle est dominée par 3 espèces: Phragmanthera usuiensis, Phragmanthera capitata et Agelanthus flammeus.

Conclusion et application des résultats: L'étude montre une forte infestation des milieux agricoles et forestiers burundais par les Loranthaceae. La lutte par suppression des touffes est peu efficace. Une stratégie de lutte plus efficace à l'échelle de tout le pays devrait être étudiée.

Mots clés : Infestation, Loranthaceae, Viscaceae, zone agro-écologique

The Loranthaceae and Viscaceae parasites of Burundi's forests and agro-ecosystems: diversity and extent of infestation

\section{ABSTRACT}

Objective: The purpose of this study was to inventory the Loranthaceaes and Viscaceae as parasites causing damages on trees in Burundi forests and agroecosystems as well as the extent of the infestation. Methodology and Results: The survey has been conducted in all agro-ecological zones of the country in forests and the surrounding agricultural areas. After prospecting, 98 sites of about 192 hectares have been 
targeted based on the abundance of infested trees. In total, 15 hemi-parasitic species have been inventoried of which 11 Loranthaceae and 4 Viscaceae. The Congo-Nile crest presents the biggest diversity with 8 species while Imbo and Kumoso count only 2 species each. Ninety five (95) host species distributed in 64 genra and 37 families have been counted of which $47 \%$ are infested by Phragmanthera usuiensis. Concerning the infestation extent, the most elevated rate is found in the depression of the Kumoso while the biggest infestation intensity is found in the depression of the Northeastern Burundi. The most elevated infestation intensities are driven by three species: Phragmanthera usuiensis, Phragmanthera capitata and Agelanthus flammeus.

Conclusion and application of results: The study shows a strong infestation of agricultural and forest ecosystems by the Loranthaceae. Fighting the parasites by Tufts cutting is not effective. A more efficient strategy should be documented in order to handle the plant parasites at national level.

Key words: infestation, Loranthaceae, Viscaceae, Agro-ecological zone

\section{INTRODUCTION}

Au Burundi, la dégradation des ressources végétales est inquiétante suite à de multiples causes: (i) le prélèvement incontrôlé des produits forestiers, (ii) le défrichement des forêts, (iii) l'exploitation minière artisanale en forêts, (iv) l'extension de l'habitat humain et autres infrastructures, (v) le surpâturage et (vi) les feux de brousse. A côté de ces causes anthropiques, la destruction des arbres et arbustes est aussi menacée par des plantes hémi-parasites appartenant aux familles des Loranthaceae et Viscaceae. Différents travaux avaient déjà mis en évidence l'existence de ces hémi-parasites dans les inventaires floristiques en forêts sans qu'ils soient considérés comme dangereux (Lewalle, 1972 ; Nzigidahera, 2000 ; Masharabu, 2012). Certains comme Phragmanthera rufescens ssp.

\section{MATÉRIEL ET MÉTHODES}

Zone d'étude : L'étude a été menée dans les 5 zones agro-écologiques du Burundi : (i) la Plaine de l'Imbo, (ii) la Crête Congo-Nil, (iii) les Plateaux centraux, (iv) la dépression du Nord-est ainsi que (v) celle du Kumoso. Ces zones présentent des caractéristiques géomorphologiques et des conditions climatiques assez variées : altitude de $774 \mathrm{~m}$ au bord du Lac Tanganyika (Plaine de l'Imbo) à $2670 \mathrm{~m}$ sur les massifs montagneux (Crête Congo-Nil), température moyenne annuelle de $15,6{ }^{\circ} \mathrm{C}$ (Imbo) à $24,1{ }^{\circ} \mathrm{C}$ (Crête CongoNil) et les précipitations de $800 \mathrm{~mm}$ à $2000 \mathrm{~mm}$. D'une zone agro-écologique à une autre, la végétation naturelle est fort variable. Les plateaux centraux qui constituent la zone agro-écologique la plus vaste du pays (52\% selon Nzigidahera 2012) connaissent aussi usuiensis, Tapinanthus constrictiflorus et Tapinanthus brunneus ssp. krausei étaient même signalés comme endémiques dans les écosystèmes naturels au Burundi (Lewalle, 1972). C'est avec leur prolifération dans les agroécosystèmes que ces hémi-parasites commencent à soulever des inquiétudes suite à leurs dégâts sur les fruitiers et autres arbres agroforestiers. L'objectif de la présente étude était donc d'inventorier les Loranthaceae et Viscaceae des différents écosystèmes, leurs hôtes et de déterminer l'ampleur d'infestation. Une stratégie de lutte contre ces hémi-parasites ne peut, en effet, être envisagée sans connaitre leur diversité et répartition aussi bien en milieu naturel que dans les agro-écosystèmes.

les plus fortes densités de population et sont donc pauvres en écosystèmes naturels.

Matériel: Le matériel végétal était composé d'échantillons des plantes des familles des Loranthaceae et Viscaceae ainsi que de leurs hôtes. Le matériel technique était composé de GPS, décamètres, appareils photo numérique, papiers journaux et pressepapier, mètres ruban, cordes, dendromètres SUUNTO et sécateurs.

\section{Méthodes}

Choix des sites et collecte des données: Dans chaque zone agro-écologique, les sites d'étude ont été choisis en milieu naturel (forêt ou savane) et dans un milieu agricole (M.A) environnant après une prospection guidée par les gardes forestiers et les 
encadreurs agricoles. Le milieu naturel (M.N) était représenté surtout par les aires protégées du fait qu'elles renferment le gros des forêts naturelles burundaises et que les Loranthaceae se retrouvaient autrefois dans les forêts. Les localités présentant de fortes infestations par les Loranthaceae et/ou Viscaceae étaient ciblées. Après prospection, 98 sites (Figure 1) faisant au total 192 ha ont été retenus. Ainsi, dans la plaine de l'Imbo, le Parc National de la Rusizi, la Réserve Naturelle forestière de Kigwena, les forêts claires de Nkayamba et Mutambara et les milieux agricoles environnant ont fait objet de l'étude. Dans cette zone, la collecte des échantillons a été réalisée dans 8 ha en milieux naturels et 12 ha en milieux agricoles, soit au total 20 hectares. Sur la Crête CongoNil, la prospection a concerné la Parc National de la Kibira (PNK), les Réserves naturelles forestières de Bururi (RNFB) et de Vyanda ainsi que les milieux agricoles riverains. Les échantillons ont été collectés dans 24 ha dont 14 en forêt et 10 en milieu agricole. Dans les plateaux centraux, le Parc national de la Ruvubu a été prospecté en Communes Mutumba de la province Karuzi et Buhinyuza de la province Muyinga. Ces lieux se sont avérés indemnes de Loranthaceae et Viscaceae. Les provinces Cankuzo, Ruyigi, Ngozi et Gitega ont été également prospectés. Au total, 85 ha ont été retenus dont seulement 8 en milieux naturels.
Dans la dépression du Nord-est, la forêt de Murehe, les milieux agricoles des communes Kirundo, Busoni, Vumbi et Ntega ont été parcourus. La récolte des échantillons a été faite sur 55 ha dont 10 en milieu forestier. Dans la dépression du Kumoso, 8 ha ont fait l'objet de l'étude dont 2 en milieux naturels.

Dans chaque hectare délimité, on procédait aux opérations suivantes :

- prise des coordonnées géographiques au GPS ;

- comptage des arbres attaqués et non attaqués ;

- pour chaque arbre attaqué : mesure de la hauteur totale, de la circonférence à hauteur de poitrine, de la circonférence des branches au niveau de la fixation du parasite, comptage du nombre de touffes de Loranthaceae/Viscaceae; identification du stade phénologique du parasite; prise de photo et récolte d'un échantillon du parasite et de son hôte pour détermination botanique et confection d'un herbier. En plus des paramètres précédents, la perception des populations locales sur les dégâts et les moyens de lutte a été documentée au moyen d'une visite de 15 ménages ciblés dans chaque commune ayant fait objet des inventaires de Loranthaceae/Viscaceae. Les travaux de terrain ont duré 4 mois, de mai à août 2013.

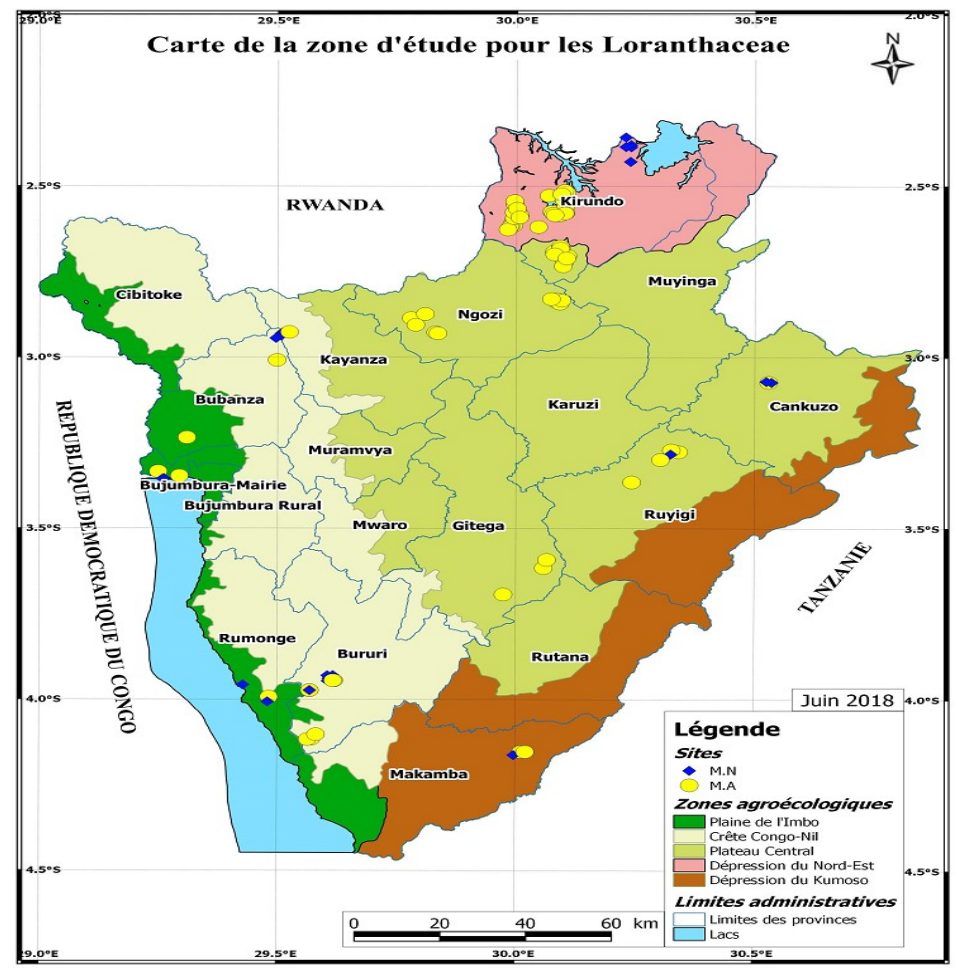

Figure 1 : Sites d'étude 
Identification des espèces parasites et leurs hôtes : Pour les espèces hôtes, une première identification a eu lieu sur terrain avec les gardes forestiers qui nous ont aidés à attribuer les noms vernaculaires. Cela ne fut pas le cas pour les Loranthaceae et Viscaceae puisque toutes les espèces fixées sur la partie aérienne d'autres plantes sont appelées « ingurukizi », littéralement « qui saute sur $»$. La détermination scientifique des échantillons a été faite à l'aide des ouvrages de Troupin : Troupin I (1978), Troupin II (1983), Troupin III (1985) et Troupin IV (1988). Pour certains échantillons, nous avons dû recourir à l'Herbarium de l'Université du Burundi et à celui de l'Office Burundais pour la protection de l'Environnement (OBPE). L'actualisation de la nomenclature des espèces parasites et hôtes inventoriées s'est référée à la base des données des plantes d'Afrique tropicale (Lebrun \& Stork, 1991-2015)

\section{RÉSULTATS ET DISCUSSION}

Diversité des Loranthaceae et Viscaceae et leur répartition dans les zones agro-écologiques: Quinze espèces d'hémi-parasites ont été identifiées: 11 de la famille des Loranthaceae et 4 de la famille des Viscaceae (Tableau 1). Parmi les Loranthaceae, Phragmanthera usuiensis est l'espèce dominante à 61, $4 \%$. Il a été retrouvé dans 4 zones écologiques sur 5 . Ce n'est pas le cas pour les autres espèces du même genre puisque Phragmanthera edouardii et $P$. capitata disponible en ligne sur le site http://www.villege.ch/musinfo/bd/cib/africal.

Traitement et analyse des données: Les données ont été compilées et traitées dans les logiciels Excel 2007 et STATA 14. Les paramètres étudiés sont: la liste des espèces de Loranthaceae et Viscaceae rencontrées, leur répartition dans les différentes zones agro-écologiques, (ii) leurs hôtes, (iii) l'ampleur d'infestation caractérisée par le taux et l'intensité d'infestation. Le taux d'infestation est le pourcentage d'individus parasités (Soro et al., 2010 ; Ondoua et al., 2015). L'intensité d'infestation ou de parasitisme est le nombre moyen de touffes d'un (des) parasite (s) par individu. Les affinités floristiques des parasites vis-àvis des espèces hôtes ont été évaluées à l'aide du logiciel MVSP 3.1. (Kovach, 1997). Le test de Chi-carré $\left(\mathrm{X}^{2}\right)$ a été appliqué pour comparer les zones agroécologiques pour le taux d'infestation.

ne se retrouvent que sur la crête Congo-Nil. Pourtant, ce dernier a été signalé comme le plus répandu au Cameroun dans la région de Douala (Dibong et al., 2008) et dans le sud de la Côte d'Ivoire (Amon et al., 2010). L'inventaire a aussi montré que, par rapport aux Loranthaceae, les Viscaceae sont très peu fréquents. Des recherches antérieures ont abouti à un résultat similaire au Népal (Joshi et Devkota 2010 ; Devokta et Kunwar, 2006).

Tableau 1 : Les espèces Loranthaceae et Viscaceae inventoriées et leur fréquence

\begin{tabular}{|l|l|l|}
\hline Espèces parasites & Fréquence & Pourcentage \\
\hline Loranthaceae & 32 & 1,27 \\
\hline Agelanthus djurensis & 12 & 0,48 \\
\hline Agelanthus flammeus & 160 & 6,37 \\
\hline Agelanthus keilii & 21 & 0,84 \\
\hline Agelanthus krausei & 54 & 2,15 \\
\hline Agelanthus musozensis & 52 & 2,07 \\
\hline Englerina woodfordiodes & 4 & 0,16 \\
\hline Eriantemum taborense & 8 & 0,32 \\
\hline Phragmanthera capitata & 62 & 2,47 \\
\hline Phragmanthera edouardii & 1540 & 61,31 \\
\hline Phragmanthera usuiensis & 338 & 13,46 \\
\hline Tapinanthus constrictiflorus & & \\
\hline Viscaceae & 15 & 0,60 \\
\hline Viscum congolense & 47 & 1,87 \\
\hline Viscum hildebrandtii & 5 & 0,20 \\
\hline Viscum triflorum & 162 & 6,45 \\
\hline Viscum tuberculatum & $\mathbf{2 5 1 2}$ & 100 \\
\hline Total & & \\
\hline
\end{tabular}


La diversité de ces hémi-parasites varie d'une zone à l'autre (Tableau 2) et aucune zone agro-écologique n'en est indemne. La crête Congo-Nil présente la plus grande diversité avec 8 espèces sur les 15, suivie des plateaux centraux (5 espèces), de la dépression du Nord-Est et de la Plaine de l'Imbo représentées chacune par 3 espèces et enfin la dépression du Kumoso (2 espèces). La grande diversité des Loranthaceae/Viscaceae sur la crête Congo-Nil peut être expliquée par le fait que par rapport aux autres zones agro-écologiques, c'est dans cette zone qu'on les retrouve sur une grande étendue de forêt. Or, selon Joshi et Devokta (2010), le milieu forestier présente une grande diversité d'espèces hôtes attirant plus d'oiseaux disséminateurs. Quant aux plateaux centraux, la diversité des hémi-parasites est probablement liée à la représentativité de cette zone en termes de superficie (52\% du territoire national). Les hémi-parasites ont été, en effet, retrouvées sur 85 ha sur les 192 inventoriées à travers toute la zone d'étude, soit $44 \%$.

Tableau 2 : Loranthaceae et Viscaceae inventoriées par zone agro-écologique du Burundi

\begin{tabular}{|c|c|c|c|c|c|}
\hline Espèces parasites & $\begin{array}{l}\text { Crête } \\
\text { Congo-Nil }\end{array}$ & $\begin{array}{l}\text { Dépression du } \\
\text { Nord-Est }\end{array}$ & $\begin{array}{l}\text { Dépression } \mathrm{du} \\
\text { Kumoso }\end{array}$ & $\begin{array}{l}\text { Plaine de } \\
\text { l'Imbo }\end{array}$ & $\begin{array}{l}\text { Plateaux } \\
\text { centraux }\end{array}$ \\
\hline \multicolumn{6}{|l|}{ Loranthaceae } \\
\hline Agelanthus djurensis & - & - & - & - & + \\
\hline Agelanthus flammeus & - & - & - & - & + \\
\hline Agelanthus keilii & - & - & + & + & + \\
\hline Agelanthus krausei & + & - & - & - & - \\
\hline Agelanthus musozensis & + & - & - & - & - \\
\hline Englerina woodfordiodes & + & - & - & - & - \\
\hline Eriantemum taborense & - & - & - & + & - \\
\hline Phragmanthera capitata & + & - & - & - & - \\
\hline Phragmanthera edouardii & + & - & - & - & - \\
\hline Phragmanthera ususiensis & + & + & + & - & + \\
\hline Tapinanthus constrictiflorus & - & - & - & + & - \\
\hline \multicolumn{6}{|l|}{ Viscaceae } \\
\hline Viscum congolense & + & - & - & - & - \\
\hline Viscum hildebrandtii & - & + & - & - & + \\
\hline Viscum triflorum & + & - & - & - & - \\
\hline Viscum tuberculatum & - & + & - & - & - \\
\hline
\end{tabular}

+ signifie présence de l'espèce, - signifie absence

Répartition des Loranthaceae et Viscaceae en milieu naturel et en milieu agricole : Certains hémiparasites se retrouvent exclusivement en milieu agricole. II s'agit d'Agelanthus djurensis et Agelanthus flammeus. D'autres comme Agelantus keilii, Agelanthus krausei, Agelanthus musozensis, Viscum congolense et
Viscum triflorum n'ont été rencontrés qu'en milieu naturel (Tableau 3). Les espèces du genre Phragmanthera se retrouvent dans les 2 types de milieux mais Phragmanthera usuiensis se retrouve à $91,5 \%$ en milieu agricole. Les viscaceae se retrouvent à $98 \%$ en milieu naturel. 
Tableau 3. Répartition du nombre d'individus attaqués par les Loranthaceae et Viscaceae selon le type de milieu

\begin{tabular}{|l|l|l|l|}
\hline Espèces parasites & Milieu agricole & Milieu naturel & Total \\
\hline Loranthaceae & 32 & & 32 \\
\hline Agelanthus djurensis & 12 & 0 & 12 \\
\hline Agelanthus flammeus & 0 & 160 & 160 \\
\hline Agelanthus keilii & 0 & 21 & 21 \\
\hline Agelanthus krausei & 0 & 54 & 54 \\
\hline Agelanthus musozensis & 12 & 40 & 52 \\
\hline Englerina woodfordiodes & 0 & 4 & 4 \\
\hline Eriantemum taborense & 4 & 4 & 8 \\
\hline Phragmanthera capitata & 19 & 43 & 62 \\
\hline Phragmanthera edouardii & 1408 & 132 & 1540 \\
\hline Phragmanthera usuiensis & 138 & 200 & 338 \\
\hline Tapinanthus constrictiflorus & & & 2283 \\
\hline Total 1 & & & 15 \\
\hline Viscaceae & 0 & 15 & 47 \\
\hline Viscum congolense & 4 & 43 & 5 \\
\hline Viscum hildebrandtii & 0 & 5 & 162 \\
\hline Viscum triflorum & 2 & 160 & 229 \\
\hline Viscum tuberculatum & & & $\mathbf{2 5 1 2}$ \\
\hline Total 2 & & & \\
\hline Total général & & & \\
\hline
\end{tabular}

Diversité des espèces hôtes : Nonante cinq espèces hôtes réparties dans 64 genres et 37 familles ont été recensées. Quarante-cinq d'entre elles, soit $47 \%$, sont infestées par Phragmanthera usuiensis et se répartissent dans 25 familles, soit $68 \%$. Cette Loranthaceae ne manifeste pas de spécificité parasitaire (Figure 2). Elle apparaît, de ce fait, comme l'espèce la plus dangereuse. Elle se retrouve avec une fréquence élevée chez Ficus thonningii (Moraceae) avec $20,59 \%$ d'individus attaqués et Persea americana
(Lauraceae) avec 15,2 \%. C'est aussi le seul parasite retrouvé chez le Caféier et le manioc. Aussi, comme ça avait été le cas dans d'autres résultats antérieurs (Joshi et Devkota 2010; Devkota et Kunwar 2006), les parasites de la famille des Viscaceae attaquent peu d'espèces $(8,4 \%)$. Le plus grand nombre d'individus de ces espèces appartiennent à Rhus longipes et Acacia hockii. Ils ont été infestés, respectivement, par Viscum tuberculatum et Viscum hildebrandtii.

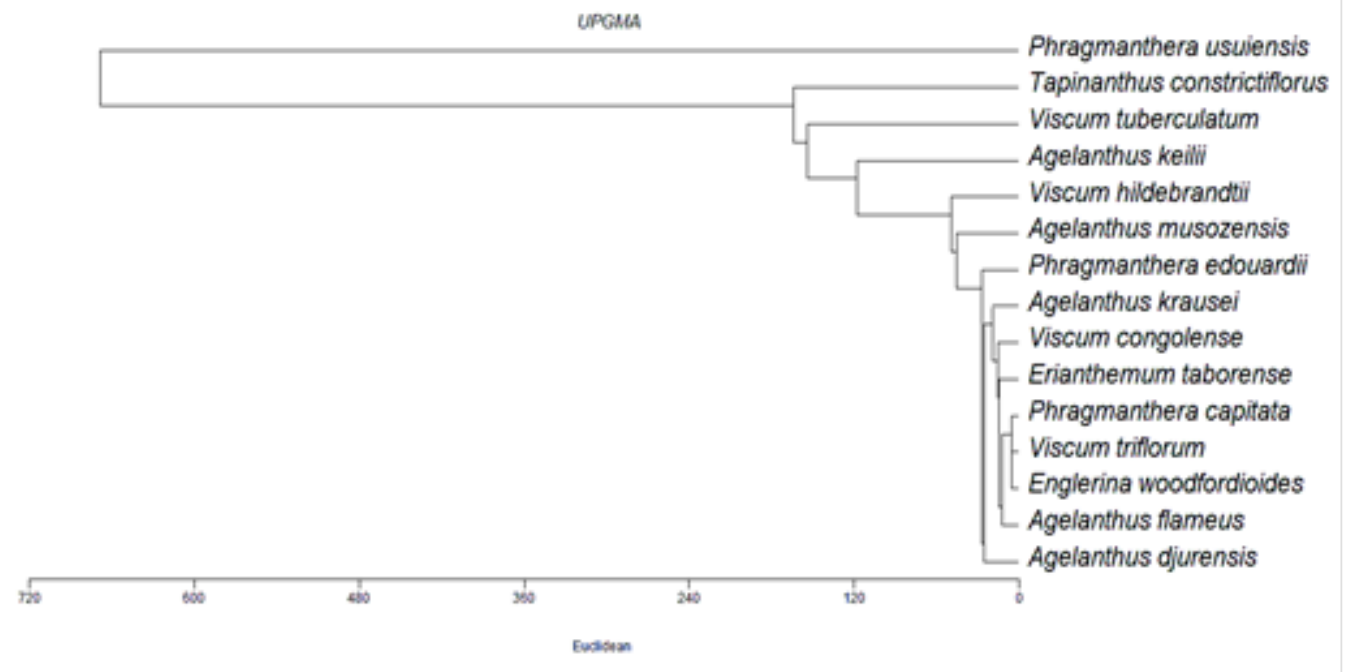

Figure 2 : Affinités floristiques des parasites vis-à-vis des espèces hôtes. 


\section{Ampleur d'infestation}

Taux d'infestation: Le taux d'infestation ou de parasitisme a été déterminé par zone agro-écologique (Figure 3) et selon le type de milieu (Tableau 4). L'analyse du taux d'infestation par zone agroécologique montre que c'est la dépression du Kumoso qui présente le taux le plus élevé (100\%). Aucun individu sain n'a été recensé dans la zone prospectée. Les plateaux centraux présentent un taux d'infestation de $67,02 \%$. La Crête Congo-Nil et la dépression du Nord-Est ont des taux respectifs de $57,63 \%$ et $53,55 \%$. C'est la plaine de l'Imbo qui a un faible taux de parasitisme $(22,38 \%)$. Le taux élevé dans la dépression du Kumoso peut être interprété avec réserves car les parasites ont été recensés sur un nombre relativement bas d'individus, soit 75 individus répartis sur une superficie de 8 ha. Cette faible densité d'arbres est normale dans les milieux agroforestiers et dans les savanes faiblement arborées de cette zone. Mais le fait que tous les individus rencontrés soient parasités reste inquiétant. Le test de Chi-carré $\left(X^{2}\right)$ montre que les différences entre zones agroécologiques sont hautement significatives $(\mathrm{Pr}=0,000)$ pour le taux d'infestation.

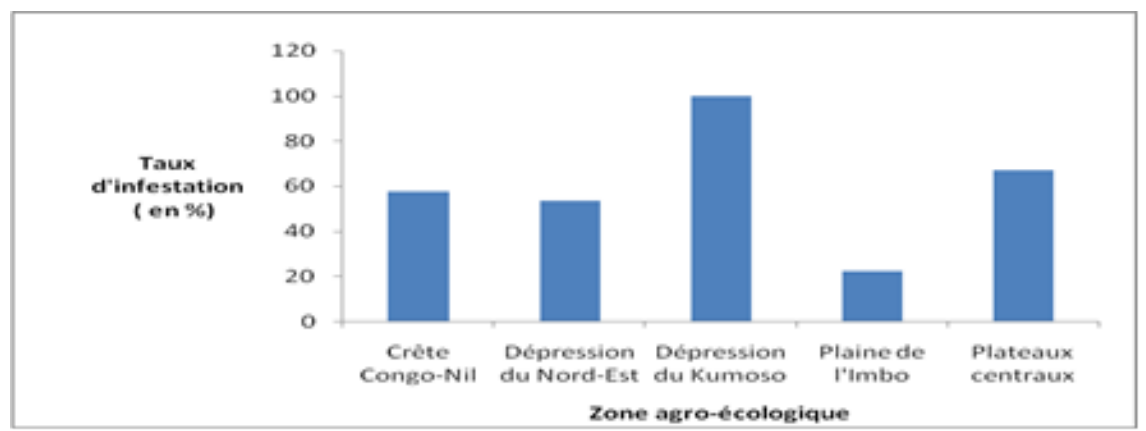

Figure 3. Taux d'infestation selon la zone agro-écologique

Concernant le type de milieu, le taux d'infestation en milieu agricole $(42,66 \%)$ est inférieur à celui du milieu naturel $(55,55 \%)$. C'est probablement lié à la densité élevée des arbres en milieu naturel.

Tableau 4 : Taux d'infestation selon le type de milieu

\begin{tabular}{l|l|l|l|l|}
\hline Type de milieu & $\begin{array}{l}\text { Nombre total } \\
\text { d'individus }\end{array}$ & $\begin{array}{l}\text { Nombre d'individus } \\
\text { parasités }\end{array}$ & $\begin{array}{l}\text { Nombre d'individus } \\
\text { sains }\end{array}$ & $\begin{array}{l}\text { Taux d'infestation } \\
(\%)\end{array}$ \\
\hline Milieu agricole & 3823 & 1631 & 2192 & 42,66 \\
\hline Milieu naturel & 1586 & 881 & 705 & 55,55 \\
\hline Total & $\mathbf{5 4 0 9}$ & $\mathbf{2 5 1 2}$ & $\mathbf{2 8 9 7}$ & \\
\hline
\end{tabular}

Intensité d'infestation: L'intensité d'infestation est le nombre moyen de touffes d'un (des) parasite (s) par individu. Elle varie selon la zone agro-écologique (Figure 4), le type de milieu (Tableau 5) et l'espèce parasite (Tableau 6). C'est la dépression du Nord-Est qui est la plus infestée avec en moyenne 8 touffes par individu, suivi des plateaux centraux ( 7 touffes par individu). Dans la dépression du Nord-est, la zone de prédilection des hémi-parasites comprend la forêt de Murehe, un écosystème fragmentée et très perturbée par les activités anthropiques et où abondent les espèces invasives comme Lantana Camara. Selon Joshi et Devkota (2010), de tels milieux sont des habitats favorables aux oiseaux disséminateurs des hémi-parasites Loranthaceae suite à la variété de ressources alimentaires. D'autre part, $30 \%$ d'espèces hôtes des milieux agricoles de cette zone sont constitués par Ficus thonningii, espèce autochtone dont le taux d'infestation est le plus élevé. 


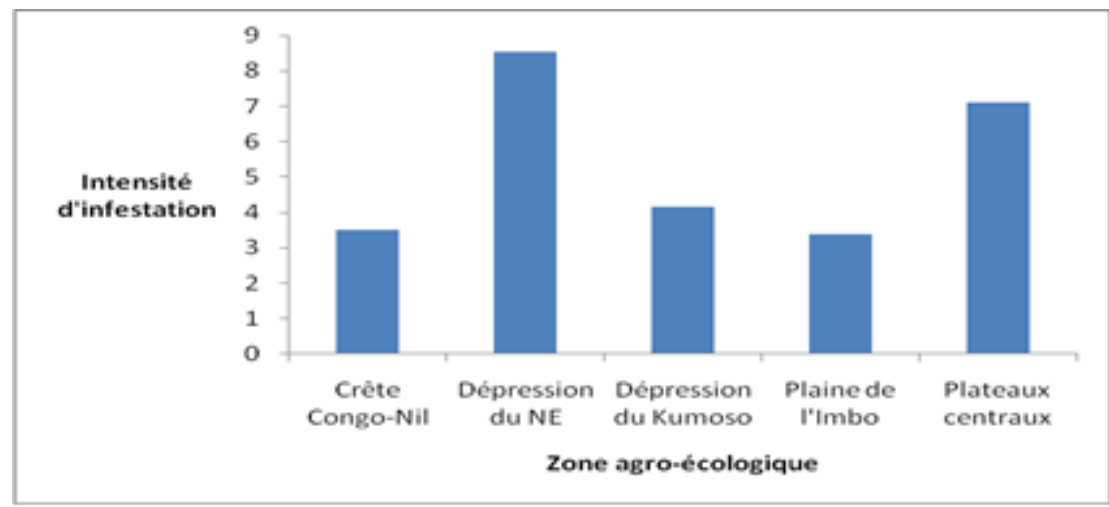

Figure 4. Intensité d'infestation selon la zone agro-écologique

Lorsqu'on considère le type de milieu, l'intensité d'infestation est plus élevée en milieu agricole $(8,1)$ par rapport au milieu naturel $(3,2)$. Ahamidé et al. (2017) avaient également trouvé chez Vittelaria paradoxa une intensité d'infestation plus élevée dans les champs $(14,76$ touffes) que dans les aires protégées $(3,62$ touffes). L'intensité d'infestation en milieu agricole burundais pourrait s'expliquer par le fait que les arbres les plus parasités sont des individus de grande taille et matures offrant aux hémi-parasites assez d'espace pour la fixation d'un grand nombre de touffes. II s'agit de Ficus thonningii, Persea americana et Grevillea robusta. Devkota et Kunwar (2006) ainsi que Ngotta et al. (2015) avaient également mis en évidence une infestation élevée liée à la taille des arbres.

Tableau 5. Intensité d'infestation selon le type de milieu

\begin{tabular}{|l|l|l|l|}
\hline Type de milieu & Nombre d'individus parasités & Nombre de touffes & Intensité d'infestation (\%) \\
\hline Milieu agricole & 1631 & 13220 & 8,1 \\
\hline Milieu naturel & 881 & 2848 & 3,2 \\
\hline
\end{tabular}

L'analyse de l'intensité d'infestation en fonction du parasite (Tableau 6) montre que les intensités les plus élevées sont dues à 3 espèces : Phragmanthera usuiensis (8,25), Phragmanthera capitata (7) et Agelanthus flammeus $(7,08)$. Le reste des espèces ont des intensités d'infestation oscillant entre 2 et 4 touffes par individus. Ces derniers résultats sont comparables à ceux trouvés au Cameroun par Massako et al. (2013) ; Massako et al. (2014) et Ondoua et al. (2015) avec des intensités d'infestations respectivement de 2,5 touffes chez le safoutier et de 4,5 touffes chez le Cacaoyier.

Tableau 6. Intensité d'infestation par parasite

\begin{tabular}{|l|l|l|l|}
\hline Espèce parasite & Nombre d'individus parasités & Nombre de touffes & Intensité d'infestation \\
\hline Agelanthus djurensis & 32 & 97 & 3,03 \\
\hline Agelanthus flammeus & 12 & 85 & 7,08 \\
\hline Agelanthus keilii & 160 & 578 & 3,61 \\
\hline Agelanthus krausei & 21 & 63 & 3,00 \\
\hline Agelanthus musozensis & 54 & 168 & 3,11 \\
\hline Englerina woodfordioides & 52 & 103 & 1,98 \\
\hline Erianthemum taborense & 4 & 14 & 3,50 \\
\hline Phragmanthera capitata & 8 & 56 & 7,00 \\
\hline Phragmanthera edouardii & 62 & 222 & 3,58 \\
\hline Phragmanthera usuiensis & 1540 & 12735 & 8,27 \\
\hline Tapinanthus constrictiflorus & 338 & 1109 & 3,28 \\
\hline Viscum congolense & 15 & 46 & 3,07 \\
\hline Viscum hildebrandtii & 47 & 153 & 3,26 \\
\hline Viscum triflorum & 5 & 11 & 2,20 \\
\hline Viscum tuberculatum & 162 & 628 & 3,88 \\
\hline
\end{tabular}


Perception des agriculteurs et méthodes de lutte : Dans les zones où les Loranthaceae abondent, les agriculteurs sont très inquiets. Très peu savent comment ces hémi-parasites sont disséminés. Certains pensent qu'ils sont apportés par le vent, d'autres pensent à un mauvais sort jeté sur leurs champs. Des diminutions des rendements chez les arbres fruitiers nous ont été signalées, surtout chez l'avocatier. Des recherches menées ailleurs en avaient également fait état (Dibong et al. 2010, Massako et al., 2013, Solikin

\section{CONCLUSION}

Cette étude aura établi une situation de base sur la présence des hémi-parasites Loranthaceae et Viscaceae ainsi que leur ampleur d'infestation au Burundi. L'inventaire dans les différentes zones agroécologiques a montré qu'il existe 11 espèces de Loranthaceae et 4 de la famille des Viscaceae. Aucune zone agro-écologique n'est indemne de ces hémiparasites. Le plus grand nombre d'espèces parasites est recensé sur la crête Congo-Nil mais le taux d'infestation le plus élevé se rencontre dans la dépression du Kumoso tandis que la dépression du Nord-Est vient en avant pour l'intensité d'infestation qui

\section{REMERCIEMENTS}

Nous remercions l'Université du Burundi pour avoir financé cette recherche ainsi que Dr. Ntukamazina

\section{RÉFÉRENCES BIBLIOGRAPHIQUES}

African Plant Database (version 3.4.0). Conservatoire et Jardin botaniques de la Ville de Genève and South African National Biodiversity Institute, Pretoria, "accès [février, 2019]", de $<$ http://www.ville-ge.ch/musinfobd/cjb/ africal>.

Ahamidé I.D.Y., Tossou M.G., Yédomonhan H. et Adomou C.A., 2017. Diversité des Loranthaceae et leur impact sur Vitellaria paradoxa C.F.Gaertn. : Un fruitier à grande valeur socio-économique au Nord-Bénin.

Amon A.D.E., Soro D., N'Guessan K et Traoré D. 2010. Les Loranthaceae: plantes vasculaires parasites des arbres et arbustes au Sud-Est de la côte d'lvoire. Journal of Applied Biosciences $25: 1565-1572$.

Boussim J.I. et Médah N., 2009. Méthodes de lutte contre les Loranthaceae. Flora et Vegetatio Sudano-Sambesica 12, 27-35.
2016). Chez l'avocatier, certains agriculteurs burundais disent que même la saveur des fruits d'arbres attaqués n'est plus bonne. La méthode de lutte en vigueur est la lutte mécanique par coupe des touffes mais l'élimination complète du parasite ne suit pas toujours. Cette méthode a pourtant donné de bons résultats sur le Karité (Boussim et Méda, 2009). Ces recherches ont démontré que la destruction du parasite par la coupe du rameau hôte en amont du point de fixation du parasite est définitive.

est en moyenne de 8 touffes par individu. Phragmanthera usuiensis s'est révélée l'espèce la plus dangereuse du fait qu'elle attaque plusieurs hôtes, surtout en milieu agricole, et que l'intensité d'infestation due à cette espèce est très élevée $(8,1)$. Les agriculteurs burundais ne savent pas encore comment lutter contre ces hémi-parasites. Comme la méthode mécanique semble la plus abordable actuellement, il est nécessaire de former les encadreurs agricoles sur le bon niveau de coupe des branches parasitées afin qu'ils puissent à leur tour aider les paysans et ainsi limiter la propagation de ces parasites.

Népomuscène pour les vérifications utiles de notre base de données.

Devkota, M.P et Kunwar, R.M, 2006. Diversity, Distribution and Host Range of Mistletoes in Godawari-Phulchoki Area, Kathmandu, Nepal. Journal of Japanese Botany 81: 255-261.

Dibong S.D., Ndongo D., Priso R.J., Taddouo V.D.n Fankem H., Salle G. et Amougou A., 2008.Parasitism of host trees by the Loranthaceae in the region of Douala (Cameroun). African journal of Environmental Science and Technology, Vol.2 (11): 371-378.

Dibong S.D., Mony R., Ndiang, Z., Ondoua, J.M., Boussim, I.J., Bilong, B. et Amougou, A., 2010. The struggle against Phragmanthera capitata (Sprengel) S. Balle (Loranthaceae) parasite of agroecosystem's fruit trees in Cameroun. Journal of Agricultural Biotechnology and Sustainable Development, vol.2 (5): 76 -81.

Joshi, G.P. and Devkota, M.P., 2010. Diversity, Host Range and Distribution of Mistletoes along Steep Altitudinal Gradient of Tribhuvan 
Highway, Central Nepal. Our Nature (2010) 8: 106-117.

Kovach W.L., 1997. MVSP: Multi-Variate Statistical Package data analysis. Kovach Computing Services; 85 Nant-y-Felin, Pentraeth, Anglesey.

Lewalle, J., 1972. Les étages de végétation du Burundi occidental. Bulletin du Jardin Botanique National de Belgique 42 (1/2) : 1-247.

Masharabu, T., 2012. Flore et végétation du Parc National de la Ruvubu au Burundi: diversité, structure et implication pour la conservation. Editions Universitaires Européennes, Sarrebruck-Allemagne, $256 \mathrm{p}$.

Massako F., Tchta M., Mony R., Yadoh Yemedah CF et Dibong S.D, 2013. Parasitisme de Dacryodes edulis par le genre Tapinanthus (Loranthaceae) et répartition de la myrmécofaune associée à Logessou Plateau (Douala, Cameroun). Journal of Applied Biosciences 68 : 5336-5346.

Massako F., Mony R., Tchta M. et Dibong S.D, 2014. Inventaire et évaluation de l'infestation des Loranthaceae sur les essences du safoutier (Dacryodes edulis (G.Don) H.J.Lam), Sciences, Technologies et Développement, volume 15, pp 87-92.

Ngotta B. J.B., Dibong, S.D., Taffouo, V.D., Ondoua, J.M. et Bilong, P., 2015. Niveau de parasitisme des hévéas par les Loranthaceae dans la région du Sud-ouest Cameroun. Journal of Applied Biosciences 96 : 9055-9062.

Nzigidahera, B., 2000. Analyse de la diversité biologique végétale nationale et identification des priorités pour sa conservation. INECN, Bujumbura, $126 \mathrm{p}$.

Nzigidahera, B., 2012. Description du Burundi : aspects physiques, Bujumbura, $10 \mathrm{p}$.

Ondoua J.P, Dibong S.D., Taffouo, V.D., Ngotta B., J.B., 2015. Parasitisme des champs semenciers de cacaoyers par les Loranthaceae dans la localité de Nkoemvone (sud Cameroun). Journal of Applied Biosciences 85 : 7794-7803.

Solikin, 2016. Population dynamic of Viscum articulatum Burm.f. on its host in Purwodadi batanic garden. Journal of Biological researches. Volume 21, $\mathrm{N}^{\circ} 2:$ 81-84.

Soro K., Soro D. N'Guessan K., Gnahoua GM et Traoré D., 2010. Parasitisme des Loranthaceae sur les hévéas en zone forestière des sous- préfectures de Gagnoa et d'Ouragahio, en Côte d'Ivoire. Journal of Animal \& Plant Sciences, Vol. 6, Issue 1: 597-604.

Troupin G., 1988. Flore du Rwanda. Spermatophytes 4. ACCT et Mus. Roy. Afr. Centr. Tervuren, Bruxelle, $651 \mathrm{p}$.

Troupin G., 1985. Flore du Rwanda. Spermatophytes 3. ACCT et INRSS Butare. $729 p$.

Troupin G., 1983. Flore du Rwanda. Spermatophytes 2. ACCT et Mus. Roy. Afr. Centr. Tervuren, Bruxelles, $603 p$.

Troupin G., 1978. Flore du Rwanda. Spermatophytes 1. Mus. Roy. Afr. Centr. Tervuren, Bruxelles, 413 p. 